TRANSACTIONS OF THE

AMERICAN MATHEMATICAL SOCIETY

Volume 356, Number 10, Pages 3985-3994

S 0002-9947(04)03451-8

Article electronically published on January 23, 2004

\title{
ON THE ASYMPTOTIC BEHAVIOR OF A COMPLETE BOUNDED MINIMAL SURFACE IN $\mathbb{R}^{3}$
}

\author{
FRANCISCO MARTÍN AND SANTIAGO MORALES
}

\begin{abstract}
In this paper we construct an example of a complete minimal disk which is properly immersed in a ball of $\mathbb{R}^{3}$.
\end{abstract}

The construction of the first complete bounded minimal surfaces, in 1996 [5], is in contrast to Calabi's conjecture that asserted the impossibility of the existence of these surfaces. When studying this family, existence questions are still important. The recent construction of surfaces of this kind with nontrivial topologies ([2, 3] has shown that this family is bigger than it actually appeared.

Nevertheless, we know that adding conditions to the boundness of a complete minimal surface leads to nonexistence theorems; for instance, 1, 7]. Besides, it has been conjectured that there does not exist a complete minimal surface which is properly immersed into the unit ball. However, a counterexample to this conjecture has been given in this paper. We have proved the following existence theorem:

Theorem 1. There exists a simply connected domain $\Omega$ of $\mathbb{C}$ and a complete minimal immersion $X: \Omega \rightarrow B$ that is proper in the open ball $B \subset \mathbb{R}^{3}$, i.e., $X(\Omega)$ has a compact intersection with any compact subset of $B$.

As in [2] and 3], the proof of this theorem is based on Nadirashvili's construction of the first complete bounded minimal surface, [5]. So, the immersion $X$ is given as the limit of a sequence of minimal immersions with boundary contained in the ball $B$, whose intrinsic metrics diverge. However, the boundaries uniformly converge to the boundary of $B$. To obtain this crucial fact we need the main lemma in paper [4] (see Lemma 2 in this paper). This behavior of the boundaries guarantees the properness of $X$.

The paper is structured as follows. In Section 2, we prove a preliminary result (Lemma 1) that we will need to obtain the main theorem. The proof of this lemma is the most difficult part of the paper. Section 3 is dedicated to proving Theorem 1

\section{Notation}

Let $X: D \rightarrow \mathbb{R}^{3}$ be a conformal minimal immersion defined on a simply connected domain $D$. If we write $X: \bar{D} \rightarrow \mathbb{R}^{3}$, it means that $X$ is defined on an open neighborhood of $\bar{D}$. Let $S=\left\{e_{1}, e_{2}, e_{3}\right\}$ be a set of orthogonal coordinates in $\mathbb{R}^{3}$. Then, label the Weierstrass representation of the minimal immersion $X$ in $S$ as

Received by the editors February 4, 2003 and, in revised form, June 20, 2003.

2000 Mathematics Subject Classification. Primary 53A10; Secondary 49Q05, 49Q10, 53C42.

Key words and phrases. Complete bounded minimal surfaces, proper minimal immersions.

This research was partially supported by MCYT-FEDER Grant no. BFM2001-3489. 
$\phi_{X}=\left\{\phi_{1}, \phi_{2}, \phi_{3}\right\}, \phi_{i}: D \rightarrow \mathbb{C}$ holomorphic functions. Recall that, in the frame $S$, the immersion $X$ can be written as follows:

$$
X(z)=\operatorname{Re}\left(\int_{z_{0}}^{z} \phi_{X}(w) d w\right)+X\left(z_{0}\right), \quad z_{0} \in D .
$$

If $G: D \rightarrow \mathbb{S}^{2}$ is the Gauss map of $X$, the meromorphic function $g=\phi_{3} /\left(\phi_{1}-i \phi_{2}\right)$ coincides with the stereographic projection of $G$. The pair $\left(g, \phi_{3}\right)$ is an alternative way of giving the Weierstrass data of the minimal immersion $X$ (see [6]). We can write the conformal metric associated to the immersion $X, \mathcal{S}_{X}=\lambda_{X}^{2}(z)\langle\cdot, \cdot\rangle$, in terms of the Weierstrass representation as follows:

$$
\lambda_{X}(z)=\frac{1}{\sqrt{2}}\left\|\phi_{X}(z)\right\|=\frac{1}{\sqrt{2}} \sqrt{\left|\phi_{1}(z)\right|^{2}+\left|\phi_{2}(z)\right|^{2}+\left|\phi_{3}(z)\right|^{2}} .
$$

Let $\alpha$ be a curve in $D$. By length $(\alpha, \mathcal{S})$ we mean the length of $\alpha$ with a metric $\mathcal{S}$. For $T \subset D$ we define the following distance: $\operatorname{dist}_{(T, \mathcal{S})}(a, b)=\inf \{\operatorname{length}(\alpha, \mathcal{S}) \mid \alpha$ : $[0,1] \rightarrow T, \alpha(0)=a, \alpha(1)=b\}$, for any $a, b \in T$. If $A$ is a subset of $T$, then $\operatorname{dist}_{(T, \mathcal{S})}(z, A)$ means the distance between point $z$ and set $A$.

Finally, by a polygon $P$ we mean a closed simple curve in $\mathbb{C}$ formed by a finite number of straight segments verifying $0 \in \operatorname{Int} P$, where $\operatorname{Int} P$ denotes the interior domain bounded by curve $P$. Given $\xi>0$ we define $P^{\xi}$ as the parallel polygonal curve in Int $P$, satisfying the following condition: that the distance between parallel sides is equal to $\xi$. Whenever we write $P^{\xi}$ in the paper we are assuming that $\xi$ is small enough to define the polygon properly.

\section{Preliminary Results}

In order to get the theorem, we deform a given minimal disk with boundary successively. In this way, we construct a sequence of minimal disks with boundary which converges to a complete bounded minimal disk, without boundary, properly immersed in a ball of $\mathbb{R}^{3}$.

The deformation that we need is given in the next lemma. Roughly speaking, we modify a minimal surface $X(\overline{\operatorname{Int} P})$ near the boundary (Property (a5)), to obtain a new minimal surface $Y(\overline{\operatorname{Int} Q})$ such that its intrinsic diameter is greater than the intrinsic diameter of $X(\overline{\operatorname{Int} P})$ (Property (a2)). At the same time, the norm of $Y$ is increased around $Q$ (Property (a3)). The norm of $Y$ is also controlled by a lower bound (Property (a4)).

Lemma 1. Let $P$ and $X: \overline{\operatorname{Int} P} \rightarrow \mathbb{R}^{3}$ be a polygon and a conformal minimal immersion, respectively, with $X(0)=0$. Consider constants $r, \varepsilon, s, a, b>0$, such that

$$
\begin{gathered}
r-a<\|X(z)\|<r, \quad \forall z \in \overline{\operatorname{Int} P \backslash \operatorname{Int} P^{\varepsilon}} ; \\
400 s+200 \varepsilon+402 a-r<0 .
\end{gathered}
$$

Then, there exists a polygon $Q$ and a conformal minimal immersion $Y: \overline{\operatorname{Int} Q} \rightarrow \mathbb{R}^{3}$, $Y(0)=0$, such that

(a1) $\overline{\operatorname{Int} P^{\varepsilon}} \subset \operatorname{Int} Q \subset \overline{\operatorname{Int} Q} \subset \operatorname{Int} P$;

(a2) $s<\operatorname{dist}_{\left(\overline{\operatorname{Int} Q}, \mathcal{S}_{Y}\right)}\left(Q, P^{\varepsilon}\right)$;

(a3) ||$|Y(z) \|-(2 R-r+2 a)|<b, \forall z \in Q$, where $R=\sqrt{r^{2}+(2 s)^{2}}+\varepsilon$;

(a4) $\|Y(z)\|>r-2 a-6(2 s+\varepsilon+2 a) r, \forall z \in \operatorname{Int} Q \backslash \operatorname{Int} P^{\varepsilon}$;

(a5) $\|Y(z)-X(z)\|<\varepsilon, \forall z \in \operatorname{Int} P^{\varepsilon}$. 
Condition (31) may seem strange. It only means than the constants $s, \varepsilon$, and $a$ must be small in terms of $r$.

Lemma 1is similar to that used by Nadirashvili in [5], but we use the machinery developed in [3] and [4]. In this way, our lemma gives more information about the norm of the immersions. This extra information is needed to prove that the immersion $X$ of Theorem 1 is proper in a ball.

Proof of Lemma 1. Consider $P$, the polygon given in the statement of Lemma 1 Our first step is to follow 3 in order to describe a labyrinth on $\operatorname{Int} P$, which depends on $P$ and a positive integer $N$.

Let $\ell$ be the number of sides of $P$. From now on $N$ will be a positive multiple of $\ell$.

Remark. Throughout the proof of the lemma a set of real positive constants $c_{1}, \ldots, c_{8}$ depending on $X, P, \rho, r, \varepsilon, s, a$, and $b$ will appear. It is important to note that the choice of these constants does not depend on the integer $N$.

Let $\zeta_{0}>0$ small enough so that $P^{\zeta_{0}}$ is well defined and $\overline{\operatorname{Int}\left(P^{\varepsilon}\right)} \subset \operatorname{Int}\left(P^{\zeta_{0}}\right)$. From now on, we will only consider $N \in \mathbb{N}$ such that $2 / N<\zeta_{0}$. Let $v_{1}, \ldots, v_{2 N}$ be a set of points in the polygon $P$ (containing the vertices of $P$ ) that divide each side of $P$ into $\frac{2 N}{\ell}$ equal parts. We can transfer this partition to the polygon $P^{2 / N}$ : $v_{1}^{\prime}, \ldots, v_{2 N}^{\prime}$. We define the following sets:

- $L_{i}=$ the segment that joins $v_{i}$ and $v_{i}^{\prime}, i=1, \ldots, 2 N$;

- $\mathcal{P}_{i}=P^{i / N^{3}}, i=0, \ldots, 2 N^{2}$

- $\mathcal{E}=\bigcup_{i=0}^{N^{2}-1} \overline{\operatorname{Int}\left(\mathcal{P}_{2 i}\right) \backslash \operatorname{Int}\left(\mathcal{P}_{2 i+1}\right)}$ and $\widetilde{\mathcal{E}}=\bigcup_{i=1}^{N^{2}} \overline{\operatorname{Int}\left(\mathcal{P}_{2 i-1}\right) \backslash \operatorname{Int}\left(\mathcal{P}_{2 i}\right)}$;

- $\mathcal{R}=\bigcup_{i=0}^{2 N^{2}} \mathcal{P}_{i}$

- $\mathcal{B}=\bigcup_{i=1}^{N} L_{2 i}$ and $\widetilde{\mathcal{B}}=\bigcup_{i=0}^{N-1} L_{2 i+1}$

- $L=\mathcal{B} \cap \mathcal{E}, \widetilde{L}=\widetilde{\mathcal{B}} \cap \widetilde{\mathcal{E}}$, and $H=\mathcal{R} \cup L \cup \widetilde{L}$;

- $\Xi_{N}=\left\{z \in \operatorname{Int}\left(\mathcal{P}_{0}\right) \backslash \operatorname{Int}\left(\mathcal{P}_{2 N^{2}}\right): \operatorname{dist}_{d s_{0}, \mathbb{C}}(z, H) \geq \frac{1}{4 N^{3}}\right\}$, where $d s_{0}$ is the Euclidean metric on $\mathbb{C}$.

We define $\omega_{i}$ as the union of the segment $L_{i}$ and those connected components of $\Xi_{N}$ that have nonempty intersection with $L_{i}$ for $i=1, \ldots, 2 N$. Finally, we label $\varpi_{i}=\left\{z \in \mathbb{C}: \operatorname{dist}_{d s_{0}, \mathbb{C}}\left(z, \omega_{i}\right)<\delta(N)\right\}$, where $\delta(N)>0$ is chosen in such a way that the sets $\overline{\varpi_{i}}(i=1, \ldots, 2 N)$ are pairwise disjoint (see Figure 1).

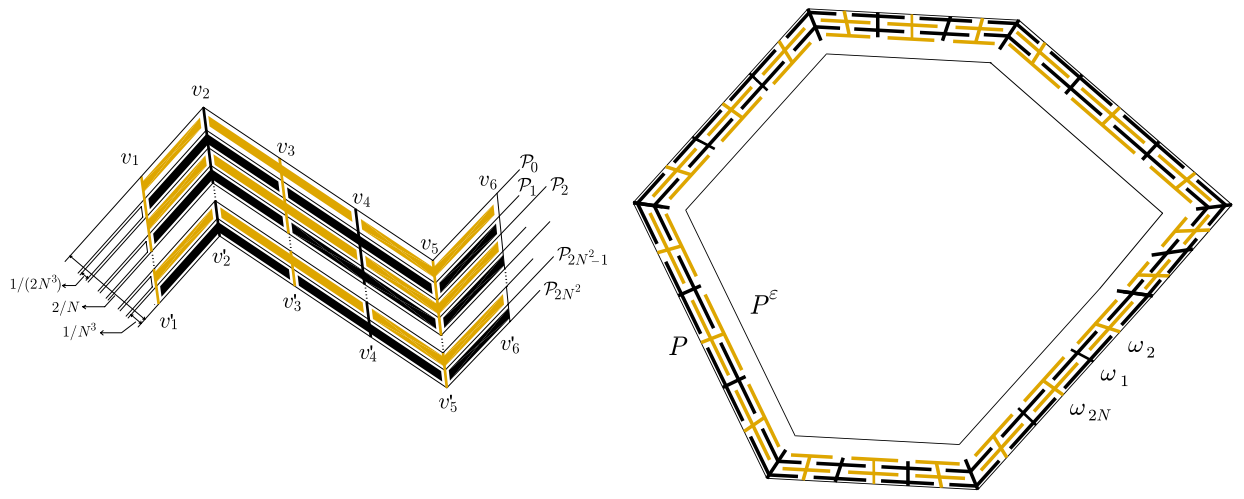

FIgURE 1. Distribution of the sets $\omega_{i}$. 
The shape of the labyrinth formed by the sets $\omega_{i}$, guarantee the following claims if $N$ is large enough:

Claim A. There is a constant $c_{1}$ such that the Euclidean diameter of $\varpi_{i}$ is less than $c_{1} / N$.

Claim B. There is a constant $c_{2}$ such that if $\lambda^{2}\langle\cdot, \cdot\rangle$ is a conformal metric on $\overline{\operatorname{Int} P}$ and verifies

$$
\lambda \geq \begin{cases}c & \text { in } \operatorname{Int} P, \\ c N^{4} & \text { in } \Xi_{N},\end{cases}
$$

for $c \in \mathbb{R}^{+}$, and if $\alpha$ is a curve in $\overline{\operatorname{Int} P}$ connecting $P^{\varepsilon}$ and $P$, then

$$
\text { length }(\alpha, \lambda\langle\cdot, \cdot\rangle)>\frac{c c_{2} N}{2} \text {. }
$$

Claim $B$ is a consequence of the fact that a curve $\alpha$, that does not go through the connected components of $\Xi_{N}$, must have a large Euclidean length.

Now, we deform $X$ in $2 N$ steps over the sets $\varpi_{1}, \ldots, \varpi_{2 N}$. Then, we construct (for a sufficiently large $N$ ) a sequence of $2 N$ conformal minimal immersions defined on $\overline{\operatorname{Int} P}: F_{0}=X, F_{1}, \ldots, F_{2 N}$, which satisfies:

$\left(\mathrm{P} 1_{i}\right) F_{i}(z)=\operatorname{Re}\left(\int_{0}^{z} \phi^{i}(w) d w\right)$;

$\left(\mathrm{P} 2_{i}\right)\left\|\phi^{i}(z)-\phi^{i-1}(z)\right\| \leq 1 / N^{2}$ for all $z \in \overline{\operatorname{Int} P} \backslash \varpi_{i}$;

$\left(\mathrm{P} 3_{i}\right)\left\|\phi^{i}(z)\right\| \geq N^{7 / 2}$ for all $z \in \omega_{i}$;

$\left(\mathrm{P} 4_{i}\right)\left\|\phi^{i}(z)\right\| \geq 1 / \sqrt{N}$ for all $z \in \varpi_{i}$;

$\left(\mathrm{P} 5_{i}\right) \operatorname{dist}_{\mathbb{S}^{2}}\left(G_{i}(z), G_{i-1}(z)\right)<\frac{1}{N \sqrt{N}}$ for all $z \in \operatorname{Int} P \backslash \varpi_{i}$, where $\operatorname{dist}_{\mathbb{S}^{2}}$ is the intrinsic distance in $\mathbb{S}^{2}$ and $G_{i}$ represents the Gauss map of the immersion $F_{i}$;

$\left(\mathrm{P} 6_{i}\right)$ there exists a set $S_{i}=\left\{e_{1}, e_{2}, e_{3}\right\}$ of orthogonal coordinates in $\mathbb{R}^{3}$ and a real constant $c_{3}>0$ such that

$\left(\mathrm{P} 6.1_{i}\right)$ if $z \in \overline{\varpi_{i}}$ and $\left\|F_{i-1}(z)\right\| \geq 1 / \sqrt{N}$, then

$$
\left\|\left(\left(F_{i-1}(z)\right)_{1},\left(F_{i-1}(z)\right)_{2}\right)\right\|<\frac{c_{3}}{\sqrt{N}}\left\|F_{i-1}(z)\right\|,
$$

$\left(\mathrm{P} 6.2_{i}\right)\left(F_{i}(z)\right)_{3}=\left(F_{i-1}(z)\right)_{3}$ for all $z \in \overline{\operatorname{Int} P}$,

where $(\cdot)_{k}$ is the $k$ th coordinate function with respect to $S_{i}$.

The sequence $F_{0}, F_{1}, \ldots, F_{2 N}$ is constructed in a recursive way.

Suppose that we have $F_{0}, \ldots, F_{j-1}$ verifying the claims $\left(\mathrm{P} 1_{i}\right), \ldots,\left(\mathrm{P} 6_{i}\right), i=$ $1, \ldots, j-1$. Then, for a large enough $N$, there are positive constants $c_{4}, \ldots, c_{7}$ such that the following statements hold:

(L1) $\left\|\phi^{j-1}\right\| \leq c_{4}$ in $\operatorname{Int} P \backslash \bigcup_{k=1}^{j-1} \varpi_{k}$.

We easily deduce this property from $\left(\mathrm{P} 2_{l}\right)$ for $l=1, \ldots, j-1$.

(L2) $\left\|\phi^{j-1}\right\| \geq c_{5}$ in Int $P \backslash \bigcup_{k=1}^{j-1} \varpi_{k}$.

To obtain this property, it suffices to apply $\left(\mathrm{P} 2_{l}\right)$ for $l=1, \ldots, j-1$ once again.

(L3) The diameter in $\mathbb{R}^{3}$ of $F_{j-1}\left(\varpi_{j}\right)$ is less than $c_{6} / N$.

This is a consequence of (L1), Claim A, and equality (1).

(L4) The diameter in $\mathbb{S}^{2}$ of $G_{j-1}\left(\varpi_{j}\right)$ is less than $c_{7} / \sqrt{N}$.

Indeed, from Claim A, the diameter of $G_{0}\left(\varpi_{j}\right)$ is bounded. (L4) holds after successive applications of $\left(\mathrm{P} 5_{l}\right)$. 
We shall now construct $F_{j}$. Taking (L3) and (L4) into account, it is not hard to find a set of orthogonal coordinates $S_{j}=\left\{e_{1}, e_{2}, e_{3}\right\}$ in $\mathbb{R}^{3}$ and a constant $c_{8}>0$ such that

(D1) if $z \in \varpi_{j}$ and $\left\|F_{j-1}(z)\right\| \geq \frac{1}{\sqrt{N}}$, then $\angle\left(e_{3}, F_{j-1}(z)\right) \leq \frac{c_{8}}{\sqrt{N}}$;

(D2) $\angle\left( \pm e_{3}, G_{j-1}(z)\right) \geq \nu / \sqrt{N}$ for all $z \in \varpi_{j}$,

where $\angle(a, b) \in\left[0, \pi\left[\right.\right.$ is the angle formed by $a$ and $b$ in $\mathbb{R}^{3}$ and $\nu$ is a constant satisfying $\nu>1 / c_{5}$. For more details see [3] and [2].

Let $\left(g^{j-1}, \phi_{3}^{j-1}\right)$ be the Weierstrass data of the immersion $F_{j-1}$ in the coordinate system $S_{j}$. Let $h_{\alpha}: \mathbb{C} \rightarrow \mathbb{C} \backslash\{0\}$ be a holomorphic function verifying:

- $\left|h_{\alpha}(z)-1\right|<1 / \alpha, \forall z \in \overline{\operatorname{Int} P} \backslash \varpi_{j}$

- $\left|h_{\alpha}(z)-1 / \alpha\right|<1 / \alpha, \forall z \in \omega_{j}$

where $\alpha$ is a sufficiently large positive number. The existence of such a function is given by Runge's theorem.

We define $\phi_{3}^{j}=\phi_{3}^{j-1}$ and $g^{j}=g^{j-1} / h_{\alpha}$. Let $F_{j}$ be the minimal immersion induced by the aforementioned Weierstrass data in the frame $S_{j}$,

$$
F_{j}(z)=\operatorname{Re}\left(\int_{0}^{z} \phi^{j}(w) d w\right) .
$$

$F_{j}$ must now verify Properties $\left(\mathrm{P} 1_{j}\right)-\left(\mathrm{P} 6_{j}\right)$. (Note that claims $\left(\mathrm{P} 1_{j}\right)-\left(\mathrm{P} 6_{j}\right)$ do not depend on changes of coordinates in $\mathbb{R}^{3}$.) Claim $\left(\mathrm{P} 1_{j}\right)$ easily holds.

Note that $h_{\alpha} \rightarrow 1$ (resp. $\left.h_{\alpha} \rightarrow \infty\right)$ uniformly on $\overline{\operatorname{Int} P} \backslash \varpi_{j}$ (resp. on $\omega_{j}$ ), as $\alpha \rightarrow \infty$. Then $\left(\mathrm{P} 2_{j}\right),\left(\mathrm{P} 3_{j}\right)$, and $\left(\mathrm{P} 5_{j}\right)$ easily hold for $\alpha$ large enough in terms of $N$.

$\left(\mathrm{P} 4_{j}\right)$ is verified using (D2) which gives

$$
\frac{\sin (\nu / \sqrt{N})}{1+\cos (\nu / \sqrt{N})} \leq\left|g^{j-1}\right| \leq \frac{\sin (\nu / \sqrt{N})}{1-\cos (\nu / \sqrt{N})} \quad \text { in } \varpi_{j},
$$

and so, taking (L2) into account one has

$$
\left\|\phi^{j}\right\| \geq\left|\phi_{3}^{j}\right|=\left|\phi_{3}^{j-1}\right| \geq \sqrt{2}\left\|\phi^{j-1}\right\| \frac{\left|g^{j-1}\right|}{1+\left|g^{j-1}\right|^{2}} \geq c_{5} \sin \left(\frac{\nu}{\sqrt{N}}\right) \geq \frac{1}{\sqrt{N}} \quad \text { in } \varpi_{j}
$$

if $N$ is large enough, which proves $\left(\mathrm{P}_{j}\right)$.

Using (D1), we get $\left(\mathrm{P} 6.1_{j}\right)$ for $c_{10}=c_{8}$. To obtain $\left(\mathrm{P} 6.2_{j}\right)$, we use $\phi_{3}^{j-1}=\phi_{3}^{j}$ in the frame $S_{j}$.

Hence, we have constructed the immersions $F_{0}, F_{1}, \ldots, F_{2 N}$ verifying claims $\left(\mathrm{P} 1_{j}\right)-\left(\mathrm{P} 6_{j}\right)$ for $j=1, \ldots, 2 N$.

In the following claim we deduce some properties of the immersion $F_{2 N}$ that are essential for our purpose.

Claim. If $N$ is large enough, then $F_{2 N}$ verifies that

(i) $2 s<\operatorname{dist}_{\left(\overline{\operatorname{Int} P}, \mathcal{S}_{F_{2 N}}\right)}\left(P, P^{\varepsilon}\right)$;

(ii) there is a $c_{9}>0$ such that $\left\|F_{j}(z)-F_{j-1}(z)\right\| \leq \frac{c_{9}}{N^{2}}, \forall z \in \operatorname{Int} P \backslash \varpi_{j}$;

(iii) $\left\|F_{2 N}(z)-X(z)\right\| \leq \frac{2 c_{9}}{N}, \forall z \in \operatorname{Int} P \backslash \bigcup_{j=1}^{2 N} \varpi_{j}$;

(iv) there is a polygon $\widetilde{P}$ such that

(iv.a) $\overline{\operatorname{Int} P^{\varepsilon}} \subset \operatorname{Int} \widetilde{P} \subset \overline{\operatorname{Int} \widetilde{P}} \subset \operatorname{Int} P$;

(iv.b) $s<\operatorname{dist}_{\left(\overline{\operatorname{Int} P}, \mathcal{S}_{F_{2 N}}\right)}\left(z, P^{\varepsilon}\right)<2 s, \forall z \in \widetilde{P}$; 
(iv.c) $\left\|F_{2 N}(z)\right\|<R, \forall z \in \overline{\operatorname{Int} \widetilde{P}}$, where $R=\sqrt{r^{2}+(2 s)^{2}}+\varepsilon$;

(iv.d) $r-2 a<\left\|F_{2 N}(z)\right\|, \forall z \in \overline{\operatorname{Int} P \backslash \operatorname{Int} P^{\varepsilon}}$.

To get Property (i) we have to apply Claim $B$ taking into account Properties $\left(\mathrm{P} 2_{i}\right),\left(\mathrm{P} 3_{i}\right)$, and $\left(\mathrm{P} 4_{i}\right)$ (see Proposition 2 in 3 for details). Properties (ii) and (iii) are deduced from $\left(\mathrm{P} 2_{i}\right)$.

We now construct the polygon $\widetilde{P}$ of Property (iv). Let

$$
\mathcal{A}=\left\{z \in \operatorname{Int} P \backslash \operatorname{Int} P^{\varepsilon}: s<\operatorname{dist}_{\left(\overline{\operatorname{nnt} P}, \mathcal{S}_{F_{2 N}}\right)}\left(z, P^{\varepsilon}\right)<2 s\right\} .
$$

$\mathcal{A}$ is a nonempty open subset of $\operatorname{Int} P \backslash \operatorname{Int} P^{\varepsilon}$. Observe that the polygons $P$ and $P^{\varepsilon}$ are contained in different arc-connected components of $\mathbb{C} \backslash \mathcal{A}$. Then, we can draw a polygonal line $\widetilde{P}$ on $\mathcal{A}$ such that $\overline{\operatorname{Int} P^{\varepsilon}} \subset \operatorname{Int} \widetilde{P} \subset \overline{\operatorname{Int} \widetilde{P}} \subset \operatorname{Int} P$.

As $\widetilde{P} \subset \mathcal{A}$, then (iv.a) and (iv.b) trivially hold.

Assertion (iv.c) follows from Properties (iv.b) and (P6 $i)$. This is now checked in the following paragraphs. Thanks to the maximum modulus theorem, we only need to prove that

$$
\left\|F_{2 N}(\eta)\right\|<R, \quad \forall \eta \in \widetilde{P} .
$$

Let $\eta \in \widetilde{P}$. If $\eta \in \operatorname{Int} P \backslash \bigcup_{j=1}^{2 N} \varpi_{j}$, we have

$$
\left\|F_{2 N}(\eta)\right\| \leq\left\|F_{2 N}(\eta)-X(\eta)\right\|+\|X(\eta)\| \leq \frac{2 c_{9}}{N}+r \leq R .
$$

On the other hand, if $\eta \in \varpi_{j}$ for $j \in\{1, \ldots, 2 N\}$, then it is slightly more complicated. From (iv.b), it is possible to find a curve $\gamma:[0,1] \rightarrow \operatorname{Int} P$ such that $\gamma(0) \in P^{\varepsilon}, \gamma(1)=\eta$, and length $\left(\gamma, \mathcal{S}_{F_{2 N}}\right) \leq 2 s$. We define

$$
\bar{t}=\sup \left\{t \in[0,1]: \gamma(t) \in \partial \varpi_{j}\right\}, \quad \bar{\eta}=\gamma(\bar{t}) .
$$

For a large enough $N$, one has $\varpi_{j} \subset \operatorname{Int}(P) \backslash \operatorname{Int}\left(P^{\varepsilon}\right)$.

To continue, we need to demonstrate that

$$
\left\|F_{j}(\bar{\eta})-F_{j}(\eta)\right\| \leq \frac{4 c_{9}}{N}+2 s .
$$

Indeed,

$$
\begin{array}{r}
\left\|F_{j}(\bar{\eta})-F_{j}(\eta)\right\| \leq\left\|F_{j}(\bar{\eta})-F_{2 N}(\bar{\eta})\right\|+\left\|F_{2 N}(\bar{\eta})-F_{2 N}(\eta)\right\|+\left\|F_{2 N}(\eta)-F_{j}(\eta)\right\| \\
\leq \frac{2 c_{9}}{N}+\operatorname{length}\left(\gamma, \mathcal{S}_{F_{2 N}}\right)+\frac{2 c_{9}}{N} \leq 4 \frac{c_{9}}{N}+2 s .
\end{array}
$$

At this point, we have two cases.

- Case 1: $\left\|F_{j-1}(\bar{\eta})\right\| \leq 1 / \sqrt{N}$. Then

$$
\begin{gathered}
\left\|F_{2 N}(\eta)\right\| \leq \| F_{2 N}(\eta)- \\
F_{j}(\eta)\|+\| F_{j}(\eta)+F_{j}(\bar{\eta})\|+\| F_{j}(\bar{\eta})-F_{j-1}(\bar{\eta})\|+\| F_{j-1}(\bar{\eta}) \| \\
\leq \frac{2 c_{9}}{N}+\frac{4 c_{9}}{N}+2 s+\frac{c_{9}}{N^{2}}+\frac{1}{\sqrt{N}} \leq R
\end{gathered}
$$

for a large enough $N$.

- Case 2: $\left\|F_{j-1}(\bar{\eta})\right\|>1 / \sqrt{N}$. In this case, from $\left(\mathrm{P} 6.2_{j}\right)$ we have (in frame $S_{j}$ )

$$
\left|\left(F_{j}(\eta)\right)_{3}\right|=\left|\left(F_{j-1}(\eta)\right)_{3}\right| \leq\left|\left(F_{j-1}(\eta)\right)_{3}-(X(\eta))_{3}\right|+\left|(X(\eta))_{3}\right| \leq \frac{2 c_{9}}{N}+r .
$$


Using inequality (44), the fact that $\bar{\eta} \in \operatorname{Int} P \backslash \varpi_{j}$, assertion (ii), and Property $\left(\mathrm{P} 6.1_{j}\right)$, one has

$$
\begin{aligned}
\left\|\left(\left(F_{j}(\eta)\right)_{1},\left(F_{j}(\eta)\right)_{2}\right)\right\| \leq & \left\|\left(\left(F_{j}(\eta)\right)_{1},\left(F_{j}(\eta)\right)_{2}\right)-\left(\left(F_{j}(\bar{\eta})\right)_{1},\left(F_{j}(\bar{\eta})\right)_{2}\right)\right\| \\
& +\left\|\left(\left(F_{j}(\bar{\eta})\right)_{1},\left(F_{j}(\bar{\eta})\right)_{2}\right)-\left(\left(F_{j-1}(\bar{\eta})\right)_{1},\left(F_{j-1}(\bar{\eta})\right)_{2}\right)\right\| \\
& +\left\|\left(\left(F_{j-1}(\bar{\eta})\right)_{1},\left(F_{j-1}(\bar{\eta})\right)_{2}\right)\right\| \\
\leq & \frac{4 c_{9}}{N}+2 s+\frac{c_{9}}{N^{2}}+\frac{c_{3}}{\sqrt{N}}\left\|F_{j-1}(\bar{\eta})\right\| \\
\leq & \frac{4 c_{9}}{N}+2 s+\frac{c_{9}}{N^{2}}+\frac{c_{3}}{\sqrt{N}}\left(\frac{2 c_{9}}{N}+r\right) \leq 2 s+\frac{c_{10}}{\sqrt{N}},
\end{aligned}
$$

where $c_{10}=4 c_{9}+c_{9}+c_{3}\left(2 c_{9}+r\right)$. By using Pythagoras' theorem, one obtains

$$
\begin{gathered}
\left\|F_{2 N}(\eta)\right\| \leq\left\|F_{2 N}(\eta)-F_{j}(\eta)\right\|+\left\|F_{j}(\eta)\right\| \\
\leq \frac{2 c_{9}}{N}+\sqrt{\left|\left(F_{j}(\eta)\right)_{3}\right|^{2}+\left\|\left(\left(F_{j}(\eta)\right)_{1},\left(F_{j}(\eta)\right)_{2}\right)\right\|^{2}}<\sqrt{r^{2}+(2 s)^{2}}+\varepsilon=R
\end{gathered}
$$

for a large enough $N$.

Finally, we check (iv.d). Let $z \in \overline{\operatorname{Int} P \backslash \operatorname{Int} P^{\varepsilon}}$. If $z \notin \bigcup_{k=1}^{2 N} \varpi_{k}$, then we deduce (iv.d) thanks to (2) and (iii). If $z \in \varpi_{j}$, we proceed as follows. We consider frame $S_{j}$, then

$$
\left\|F_{2 N}(z)\right\| \geq\left\|F_{j}(z)\right\|-\frac{2 c_{9}}{N} \geq\left|\left(F_{j}(z)\right)_{3}\right|-\frac{2 c_{9}}{N}=\left|\left(F_{j-1}(z)\right)_{3}\right|-\frac{2 c_{9}}{N} \geq
$$

Now, we use $\left(\mathrm{P} 6.1_{j}\right)$ (notice that $\left\|F_{j-1}(z)\right\|>1 / \sqrt{N}$ from (2) )):

$$
\geq\left\|F_{j-1}(z)\right\|\left(1-\frac{c_{3}}{\sqrt{N}}\right)-\frac{2 c_{9}}{N}>\left(r-a-\frac{2 c_{9}}{N}\right)\left(1-\frac{c_{3}}{\sqrt{N}}\right)-\frac{2 c_{9}}{N}>r-2 a,
$$

for large enough $N$.

This completes the proof of the claim.

The immersion $F_{2 N}$ and the polygon $\widetilde{P}$ verify all the properties of Lemma [1] except claim (a3). In order to get this property, we modify $F_{2 N}$ by using the following lemma:

Lemma $2([4])$. Let $\hat{X}: \hat{O} \longrightarrow \mathbb{R}^{3}$ be a conformal minimal immersion defined on a simply connected domain $\hat{O}$ with $\hat{X}(0)=0$. Consider $\hat{r}>0,0<\hat{s}<\hat{r} / 100$ and a polygon $\hat{P}$ with $\hat{P} \subset \hat{O}$, satisfying

$$
\hat{r}<\|\hat{X}(z)\|<\hat{r}+\hat{s} / 2, \quad \forall z \in \hat{O} \backslash \operatorname{Int} \hat{P} .
$$

Then, for any $\hat{b}_{1}, \hat{b}_{2}>0$, there exist a polygon $Q$ and a conformal minimal immersion $Y: U \longrightarrow \mathbb{R}^{3}$ defined on an open neighborhood of $\overline{\operatorname{Int} Q}$ with $Y(0)=0$, such that

(b1) $\hat{P} \subset \operatorname{Int} Q \subset \overline{\operatorname{Int} Q} \subset U \subset \hat{O}$;

(b2) $\|Y(z)-\hat{X}(z)\|<\hat{b}_{1}, \forall z \in \overline{\operatorname{Int} \hat{P}}$;

(b3) ||$|Y(z) \|-(\hat{r}+\hat{s})|<\hat{b}_{2}, \forall z \in Q$;

(b4) $\|Y(z)\|>\hat{r}-3 \sqrt{\hat{s} \hat{r}}, \forall z \in \operatorname{Int} Q \backslash \operatorname{Int} \hat{P}$.

This lemma allows us to modify the minimal surface $F_{2 N}$ near the boundary (Property (b2)), to obtain a new minimal surface $Y$ such that the norm of $Y$ along a polygon increases with respect to the norm of $F_{2 N}$ (Property (b3)). At the same time, the norm of $Y$ is controlled by a lower bound (Property (b4)). 
Hence, apply the lemma to the following data:

$\hat{X}=F_{2 N}, \quad \hat{P}=\widetilde{P}, \quad \hat{r}=r-2 a, \quad \hat{s}=2 R-2 r+4 a, \quad \hat{b}_{2}=b$, and

$\hat{O} \subset \operatorname{Int} P$ is an open neighborhood of $\overline{\operatorname{Int} \widetilde{P}}$ which is small enough to verify (5). The constant $\hat{b}_{1}$ is chosen small enough, as we will see below.

Here, Properties (iv.c), (iv.d), and (3) guarantee that the data satisfy the hypothesis of the lemma. Then, we consider a polygon $Q$ and a minimal immersion $Y: \overline{\operatorname{Int} Q} \rightarrow$ $\mathbb{R}^{3}$ given by applying Lemma 2 to the above data. It is easy to check that $Y$ and $Q$ verify all the claims in Lemma 1. Notwithstanding, we need to clarify claims (a2) and (a4).

Claim (a2) is a consequence of (iv.b) and the fact that $Y \rightarrow F_{2 N}$, uniformly on $\overline{\operatorname{Int} \widetilde{P}}$, as $\hat{b}_{1} \rightarrow 0$.

Claim (a4) is deduced as follows: If $z \in \operatorname{Int} Q \backslash \operatorname{Int} \widetilde{P}$, from (b4) of Lemma 2, we deduce $\|Y(z)\|>r-2 a-6(2 s+\varepsilon+2 a) r$. On the other hand, if $z \in \operatorname{Int} \widetilde{P} \backslash \operatorname{Int} P^{\varepsilon}$, using (iv.d) and (b2) of Lemma 2 we deduce $\|Y(z)\|>\left\|F_{2 N}(z)\right\|-1 / N>r-2 a-$ $1 / N$. This completes the proof of Lemma 1.

\section{Proof of Theorem 1}

We use Lemma 1 to construct a sequence

$$
\chi_{n}=\left(X_{n}: \overline{\operatorname{Int} P_{n}} \rightarrow \mathbb{R}^{3}, P_{n}, \varepsilon_{n}, \xi_{n}, r_{n}, \rho_{n}\right),
$$

where $X_{n}$ are conformal minimal immersions with $X_{n}(0)=0, P_{n}$ are polygons, and $\left\{\varepsilon_{n}\right\},\left\{\xi_{n}\right\},\left\{r_{n}\right\},\left\{\rho_{n}\right\}$ are sequences of positive numbers verifying

- $\varepsilon_{n}<1 / n^{3}$

- $\left\{\xi_{n}\right\} \searrow 0$

- $r_{1}>402$ and $r_{n}=2 \sqrt{r_{n-1}^{2}+(2 / n)^{2}}+2 \varepsilon_{n}-r_{n-1}+2 / n^{2}+1 / n^{3}$;

- $\rho_{1}>0$, and $\rho_{n}=\rho_{1}+\sum_{i=2}^{n} 1 / i$ for $n \geq 2$.

The sequence $\left\{\chi_{n}\right\}$ must verify the following properties:

$\left(\mathrm{A}_{n}\right) \rho_{n}<\operatorname{dist} \frac{}{\left(\overline{\operatorname{Int} P P_{n}^{\xi_{n}}}, \mathcal{S}_{X_{n}}\right)}\left(0, P_{n}^{\xi_{n}}\right)$;

$\left(\mathrm{B}_{n}\right)\left\|X_{n}(z)-X_{n-1}(z)\right\|<\varepsilon_{n}, \forall z \in \operatorname{Int} P_{n-1}^{\xi_{n-1}}$;

$\left(\mathrm{C}_{n}\right) \lambda_{X_{n}}(z) \geq \alpha_{n} \lambda_{X_{n-1}}(z), \forall z \in \operatorname{Int} P_{n-1}^{\xi_{n-1}}$, where $\left\{\alpha_{i}\right\}_{i \in \mathbb{N}}$ is a sequence of real numbers such that $0<\alpha_{i}<1$ and $\left\{\prod_{i=1}^{n} \alpha_{i}\right\}_{n}$ converges to $1 / 2$;

$\left(\mathrm{D}_{n}\right) \overline{\operatorname{Int} P_{n-1}^{\xi_{n-1}}} \subset \operatorname{Int} P_{n-1}^{\varepsilon_{n}} \subset \overline{\operatorname{Int} P_{n-1}^{\varepsilon_{n}}} \subset \operatorname{Int} P_{n}^{\xi_{n}} \subset \overline{\operatorname{Int} P_{n}^{\xi_{n}}} \subset \operatorname{Int} P_{n} \subset \overline{\operatorname{Int} P_{n}} \subset$ Int $P_{n-1}$;

$\left(\mathrm{E}_{n}\right) r_{n}-\frac{1}{(n+1)^{2}}<\left\|X_{n}(z)\right\|<r_{n}, \forall z \in P_{n}$;

$\left(\mathrm{F}_{n}\right) r_{n-1}-\frac{2}{n^{2}}-\frac{30 r_{n-1}}{n}<\left\|X_{n}(z)\right\|, \forall z \in \operatorname{Int} P_{n} \backslash \operatorname{Int} P_{n-1}^{\varepsilon_{n}}$.

The sequence $\left\{\chi_{n}\right\}$ is constructed in a recursive way. To define $\chi_{1}$, we can take $X_{1}$ as a plane, and then choose the rest of the elements.

Suppose that we have $\chi_{1}, \ldots, \chi_{n}$. Consider the following data:

$$
X=X_{n}, \quad P=P_{n}, \quad a=\frac{1}{(n+1)^{2}}, \quad \rho=\rho_{n}, \quad r=r_{n}, \quad s=\frac{1}{(n+1)} .
$$

Note that $\left\{r_{n}\right\}$ is an increasing sequence and $r_{1}>400 s+402 a$, so $400 s+402 a-$ $r_{n}<0$. Furthermore, Property $\left(\mathrm{E}_{n}\right)$ tells us that $r-a<\|X\|<r$ on $P$. Then it is straightforward that we can find a small enough positive constant $\kappa$, such that Lemma 1 can be applied to the aforementioned data, and $0<\varepsilon<\kappa$ and $0<b$. 
Take a sequence $\left\{\widehat{\varepsilon}_{m}\right\} \searrow 0$, with $\widehat{\varepsilon}_{m}<\operatorname{minimum}\left\{\frac{1}{(n+1)^{3}}, \kappa\right\}, \forall m$. For each $m$, we consider $Q_{m}$ and $Y_{m}: \overline{\operatorname{Int} Q_{m}} \rightarrow \mathbb{R}^{3}$ given by Lemma 1 , for the above data and $\varepsilon=\widehat{\varepsilon}_{m}$ and $b=\widehat{\varepsilon}_{m}$. If $m$ is large enough, assertions (a1) and (a5) in Lemma [1 tell us that $\overline{\operatorname{Int} P_{n}^{\xi_{n}}} \subset \operatorname{Int} Q_{m}$ and the sequence $\left\{Y_{m}\right\}$ converges to $X_{n}$ uniformly in $\overline{\operatorname{Int} P_{n}^{\xi_{n}}}$. In particular, $\left\{\lambda_{Y_{m}}\right\}$ converges uniformly to $\lambda_{X_{n}}$ in $\overline{\operatorname{Int} P_{n}^{\xi_{n}}}$. Therefore, there is a $m_{0} \in \mathbb{N}$ such that

$$
\begin{array}{rcl}
\overline{\operatorname{Int} P_{n}^{\xi_{n}}} \subset & \operatorname{Int} P_{n}^{\hat{\varepsilon}_{m_{0}}} & \subset \operatorname{Int} Q_{m_{0}}, \\
\rho_{n} & < & \operatorname{dist}\left(\overline{\operatorname{Int} P_{n}^{\xi_{n}}}, \mathcal{S}_{Y_{m_{0}}}\right) \\
\lambda_{Y_{m_{0}}} & \geq & \alpha_{n+1} \lambda_{X_{n}} \quad \text { in } \operatorname{Int} P_{n}^{\xi_{n}} .
\end{array}
$$

We define $X_{n+1}=Y_{m_{0}}, P_{n+1}=Q_{m_{0}}$, and $\varepsilon_{n+1}=\widehat{\varepsilon}_{m_{0}}$. From (6), (7) and statement (a2) in Lemma 1, it is not hard to see that $\rho_{n+1}<\operatorname{dist}_{\left(\overline{\operatorname{Int} P_{n+1}}, \mathcal{S}_{X_{n+1}}\right)}\left(0, P_{n+1}\right)$. Finally, take $\xi_{n+1}$ small enough such that $\left(\mathrm{A}_{n+1}\right)$ and $\left(\mathrm{D}_{n+1}\right)$ hold.

$\left(\mathrm{E}_{n+1}\right)$ is a direct consequence of statement (a3) in Lemma 1 the definition of $r_{n+1}$ and the fact that $\varepsilon_{n+1}<1 /(n+1)^{3}$.

In order to check $\left(\mathrm{F}_{n+1}\right)$, consider $z \in \operatorname{Int} P_{n+1} \backslash \operatorname{Int} P_{n}^{\varepsilon_{n+1}}$. Then, we use (a4) to obtain

$$
\begin{aligned}
& \left\|X_{n+1}(z)\right\|>r_{n}-\frac{2}{(n+1)^{2}}-6\left(\frac{2}{n+1}+\varepsilon_{n+1}+\frac{2}{(n+1)^{2}}\right) r_{n} \\
& \quad=r_{n}-\frac{2}{(n+1)^{2}}-\frac{6}{n+1}\left(2+\varepsilon_{n+1}(n+1)+\frac{2}{n+1}\right) r_{n}>r_{n}-\frac{2}{(n+1)^{2}}-30 \frac{r_{n}}{n+1} .
\end{aligned}
$$

The remaining properties directly follow from (6), (8) and the aforementioned lemma. This concludes the construction of the sequence $\left\{\chi_{n}\right\}_{n \in \mathbb{N}}$.

Now, we extract some information from the properties of $\left\{\chi_{n}\right\}$. First, from $\left(\mathrm{B}_{n}\right)$, we deduce that $\left\{X_{n}\right\}$ is a Cauchy sequence, uniformly on compact sets of $\Omega=\bigcup_{n}$ Int $P_{n}^{\xi_{n}}$, and so $\left\{X_{n}\right\}$ converges on $\Omega$. If one employs Properties $\left(\mathrm{D}_{n}\right)$, then the set $\Omega$ is an expansive union of simply connected domains, resulting in $\Omega$ being simply connected. Let $X: \Omega \rightarrow \mathbb{R}^{3}$ be the limit of $\left\{X_{n}\right\}$. Then $X$ has the following properties:

- $X$ is a conformal minimal immersion (Properties $\left(\mathrm{C}_{n}\right)$ );

- $\Omega$ is complete with the metric $\mathcal{S}_{X}$ (Properties $\left(\mathrm{A}_{n}\right)$ and $\left.\left(\mathrm{C}_{n}\right)\right)$;

- Observe that $\lim r_{n}=\sigma \in \mathbb{R}$. Then $X: \Omega \rightarrow B_{\sigma}$ is proper in the ball $B_{\sigma}=\left\{p \in \mathbb{R}^{3}:\|p\|<\sigma\right\}$ (Properties $\left(E_{n}\right)$ and $\left(F_{n}\right)$ ).

For further details about this proof, see [2], 3], [4], and [5].

Remark 1. Our method of construction provides examples of complete, properly immersed minimal surfaces in a ball, but without any kind of control on the image in the sphere of the radial limit points of the immersion. It would be interesting to study this limit set. For instance, is the limit set dense? or can it omit an open domain of the sphere?

\section{REFERENCES}

[1] D. Hoffman, W. H. Meeks III, The strong halfspace theorem for minimal surfaces. Invent. Math. 101 (1990), no. 2, 373-377. MR 92e:53010

[2] F. J. López, F. Martín, and S. Morales, Adding handles to Nadirashvili's surfaces. J. Differential Geom. 60 (2002), no. 1, 155-175. MR 2003f:53013 
[3] F. Martín and S. Morales, A complete bounded minimal cylinder in $\mathbb{R}^{3}$. Michigan. Math. J. 47 (2000), 499-514. MR 2001m:53015

[4] S. Morales, On the existence of a proper minimal surface in $\mathbb{R}^{3}$ with the conformal type of a disk. G.A.F.A., to appear.

[5] N. Nadirashvili, Hadamard's and Calabi-Yau's conjectures on negatively curved and minimal surfaces. Invent. Math. 126 (1996), 457-465. MR 98d:53014

[6] R. Osserman, A survey of minimal surfaces. Dover, New York, 1986. MR 87j:53012

[7] F. Xavier, Convex hulls of complete minimal surfaces. Math. Ann. 269 (1984), no. 2, 179-182. MR 86c:53006

Departamento de Geometría y Topología, Universidad de Granada, 18071 Granada, SPAIN

E-mail address: fmartin@ugr.es

Departamento de Geometría y Topología, Universidad de Granada, 18071 Granada, SPAIN

E-mail address: santimo@ugr.es 